\title{
Preparing for imported Ebola cases in Israel, 2014 to 2015
}

T Brosh-Nissimov ${ }^{12}$, L Poles ${ }^{13}$, M Kassirer ${ }^{12}$, R Singer ${ }^{4}$, E Kaliner ${ }^{14}$, DD Shriki ${ }^{4}$, E Anis ${ }^{14}$, I Fogel ${ }^{12}$, D Engelhard ${ }^{15}$, I Grotto ${ }^{14}$ , on behalf of the Israeli Epidemic Management Team ${ }^{1}$

1. Epidemic Management Team, Ministry of Health, Israel

2. CBRN Medicine Branch, Israel Defense Forces Medical Corps, Israel

3. Kaplan Medical Center, Rehovot, Israel

4. Public Health Services, Ministry of Health, Jerusalem, Israel

5. Pediatric Division, Hadassah-Hebrew University Medical Center, Ein Kerem, Jerusalem, Israel

Correspondence: Tal Brosh-Nissimov (tbrosh@gmail.com)

Citation style for this article:

Brosh-Nissimov T, Poles L, Kassirer M, Singer R, Kaliner E, Shriki DD, Anis E, Fogel I, Engelhard D, Grotto I. Preparing for imported Ebola cases in Israel, 2014 to 2015. Euro Surveill. 2015;20(44):pii=30054. DOI: http://dx.doi.org/10.2807/1560-7917.ES.2015.20.44.30054

Article submitted on 25 June 2015 / accepted on 14 October 2015 / published on 05 November 2015

During the current outbreak of Ebola virus disease (EVD) in West Africa, preventing exportation of the disease posed many challenges for economically more developed countries. In Israel, although the risk of importing single cases was assumed to be low, the implications of local transmission were great. This article describes the EVD preparedness plan of the Israeli Ministry of Health. Key elements were a sensitive case definition, designation of a single treatment centre for suspected and confirmed cases, construction of a mobile unit using customised negative-pressure tents and a vigorous national training programme. There were no patients with EVD in Israel, but a few suspected cases were assessed. The Israeli plan may provide a template for emergency infectious disease response in other geographically small countries.

\section{Introduction}

The recent epidemic of Ebola virus disease (EVD), beginning in late 2013 in West Africa, was the largest ever reported, with a case count of over 28,500 and more than 11,000 deaths until 28 October 2015 [1]. The vast majority of cases were concentrated in three West African countries, with only 22 cases exported to or presented at eight European countries and the United States (US) [2-8]. However, the failure of local medical systems to control the outbreak and the presence of foreign medical teams in the affected countries raised concerns about exportations to other countries among the public, healthcare professionals and government authorities. These fears increased following reports of three cases of nosocomial transmission in the US and in Spain $[4,9]$. Many efforts and resources were invested worldwide to prepare for EVD exportation.

This report describes the national preparedness plan for EVD in Israel, with its unique characteristics and solutions.

\section{Response planning}

The Israeli preparedness plan for EVD was developed and executed by the Israeli Ministry of Health $(\mathrm{MOH})$, with advice from the Epidemic Management Team (EMT), a multi-disciplinary task force supporting decision making about biothreats comprising members from every relevant health profession and other organisations. The EMT adheres to the international guidelines of the World Health Organization (WHO), the Centers for Disease Control and Prevention (CDC) in the US and the European Centre for Disease Prevention and Control (ECDC). Published lessons, data and cases from West Africa were tracked, and expert teams were appointed to advise the EMT on specific problems. The $\mathrm{MOH}$ rapidly issued regulations granting its Director General special powers in dealing with potential EVD cases, including the right to enforce examination and to monitor, isolate, quarantine and treat suspected patients and their contacts.

Media communication was coordinated proactively by the $\mathrm{MOH}$ headquarters. Overall, the media and the public were accepting of the government measures.

\section{Risk assessment}

An assessment of the potential for international spread of EVD through routine travel from the three affected countries estimated an average of 2.8 exported cases globally per month [10]. Given that there was little direct communication and no direct flight routes between Israel and the countries affected by EVD, the probability of cases originating from returning citizens or travelling foreigners was assumed to be very low. A higher risk was anticipated were the epidemic to spread to other African countries that have closer ties with Israel. The possibility of disease in an Israeli healthcare worker (HCW) returning from an Ebolaaffected area was considered, but no restrictions were 


\section{FIGURE 1}

The mobile IsoArk, an isolation unit used in the Ebola Treatment Center, Israel, 2015 A

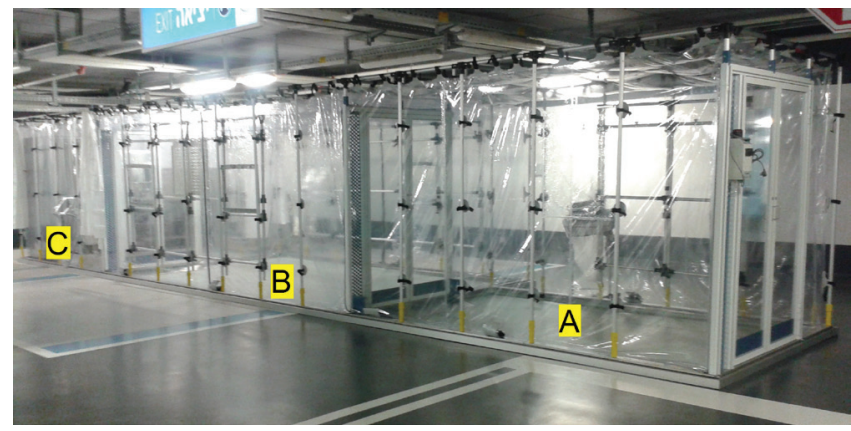

The unit contains three separate chambers with increasing negative pressure and unidirectional airflow.

A. Wide anteroom for the passage of staff and equipment and doffing of personal protective equipment.

B. Main treatment area, size: $5.3 \times 3.2 \mathrm{~m}$, equipped as an intensive care unit.

C. Shower, toilet and waste treatment unit

placed on Israeli volunteers. Very few Israeli volunteer HCWs reported treating of EVD patients directly, and an estimated 50 Israeli aid workers participated in logistical and psychosocial support missions without direct contact with patients.

Even in the three affected countries, with poor hygienic conditions and healthcare infrastructure, the reported level of contagiousness of EVD was not high, with mean basic reproduction rates (Ro) ranging from 1.71 to 2.02 in Guinea, Sierra Leone and Liberia [11]. Therefore, a very limited number of secondary cases was expected, especially if stringent infection control protocols and active surveillance of incoming travellers were implemented. With these considerations, the $\mathrm{MOH}$ directive was designed to prepare for up to four patients with EVD simultaneously (one or two imported patients and up to two secondary cases).

\section{Case definition}

Case definitions of suspected EVD developed over time and varied by country $[12,13]$. Owing to the small number of potential cases expected in Israel, a relatively sensitive suspected case definition was selected: any person who presented with a fever of $38^{\circ} \mathrm{C}$ or more and had visited a country with widespread EVD transmission within 21 days before symptom onset [14]. Confirmed cases were defined by a positive PCR test for Ebola virus. Suspected cases were designated as such only on approval of the public health services (PHS), mandating early national involvement in every single case. Entry screening was implemented, with 21-day follow-up for all travellers arriving from endemic countries. The $\mathrm{MOH}$ guidelines called for the

\section{FIGURE 2}

Bathroom and toilet facilities inside the IsoArk isolation unit

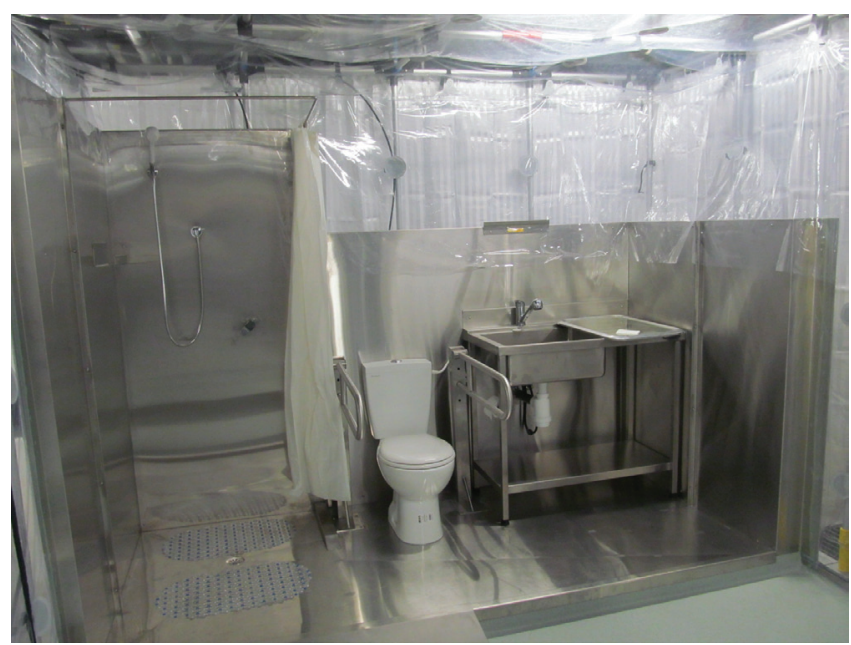

All liquid waste is actively pumped into a decontamination system located outside the tent. exclusion of other common causes of fever in returning travellers, especially life-threatening diseases such as malaria. This was to be done only in the designated Ebola centre.

\section{Infection control}

The principle route of EVD transmission is contact with contaminated body fluids, primarily vomit, faeces and blood $[15,16]$. Epidemiological data do not support airborne transmission, and secondary cases have been described almost exclusively among household contacts and persons in direct contact with patients. Nevertheless, it was argued by some that airborne transmission may occur [17], and the WHO and the CDC recommend that $\mathrm{HCW}$ apply airborne precautions during aerosol-generating procedures such as intubation and suctioning, and in events of spillage of contaminated excretions $[15,18]$. A small number of animal studies have suggested airborne pig-to-primate and primate-to-primate transmission $[19,20]$. Furthermore, owing to their stability in aerosols, filoviruses are considered a potential category A biological weapon [2123]. Therefore, the $\mathrm{MOH}$ incorporated the universal recommendations for airborne precautions, including placement of the patient in negative-pressure isolation rooms, and use of $\mathrm{N}_{95}\left(\mathrm{FFP}_{3}\right)$ respirators by caregivers, simplifying protection recommendations and avoiding accidental airborne exposures.

Personal protective equipment (PPE) for caregivers was specified and purchased centrally by the MOH and distributed to all acute care hospitals in the country and other relevant caregivers. The overall cost for PPE and additional supplies for infection control was estimated 
as EUR 1 million. The standard PPE kit for all HCWs includes hospital scrubs, rubber boots, water-resistant coveralls (EN 14126 biological protection standard), water-resistant shoe covers, two pairs of nitrile gloves, an N95 respirator, a hood to cover exposed areas of the head and a face shield. In addition, the $\mathrm{MOH}$ recommended the use of a water-impermeable coverall under conditions of heavy exposure to liquid spillage, such as bathing the patient or cleaning the room. The ministry also prescribed a specific universal donning and doffing procedure [14]. Safe doffing was ensured by co-worker supervision and rinsing of gloved hands with bleach at each stage.

Although internal medicine departments in Israeli hospitals have isolation rooms, these were considered inappropriate for patients with EVD because they did not allow for optimal spatial separation from the surrounding rooms, precluding care of non-EVD patients in the same department. Furthermore, although Israeli public knowledge about EVD was reported to be quite good [24], hospital administrators were very reluctant to designate these rooms for EVD patients for fear of stigmatising entire wards or even buildings. Such perceptions, although scientifically unsubstantiated, are not uncommon in infectious disease outbreaks [25]. Therefore, a portable, modular, free-standing isolation unit was constructed using customised negativepressure tents (IsoArk, Beth-El Industries Ltd, Zikhron Yaakov, Israel). The tent, originally designed in 2003 for the isolation of patients infected with the airborne severe acute respiratory syndrome (SARS) virus, contains an anteroom for safe passage of staff and equipment and a room used as an intensive care unit. In order to adapt the unit to EVD patients, a 'wet' room with running water and a toilet was added. Airflow is unidirectional, and an external decontamination system inactivates drained liquid waste (Figure 1 and Figure 2).

\section{Allocation of roles within the Israeli health system}

The preparedness plan involved all components of the Israeli health system. Because of the limited time available to train staff and ensure the highest infection control standards, the $\mathrm{MOH}$ designated a single hospital to serve as the national Ebola treatment centre (ETC). Rambam Medical Center in Haifa was chosen because of its excellent infection control practices and a large emergency infrastructure, which supported the construction of isolation facilities outside its main campus. An underground complex that is intended to serve as an all-hazards emergency hospital was selected as the optimal site, to be staffed by volunteers from various hospital departments. The ETC was eventually housed in free-standing IsoArk units, containing patient rooms and an on-site laboratory. Provisions were made to support intensive care, including the spacious patient rooms $(5.3 \times 3.2 \mathrm{~m})$, necessary equipment for respiratory and haemodynamic support and intensive care staff. Renal support could theoretically be provided by plasmapheresis. Laboratory capabilities included blood count, basic chemistry, blood gas analysis and coagulation tests. Imaging was based on a portable X-ray unit inserted through a dedicated plastic sleeve near the patient's bed and a digital cassette wrapped in plastic bags. Separated sections were allocated for donning and doffing and provisions were made for autoclaving of all solid waste. Patients thus had access to tertiary level care without exposing general hospital facilities to potential Ebola virus contamination.

All other hospital emergency departments and community clinics were instructed to provide only life-saving care for suspected cases while adhering to strict isolation and personal protection practices as long as the patient was under their care. They were to immediately report the case to the district public health officer, followed by transfer to the ETC, without performing any laboratory or imaging work-up [14]. This delay in patient care was considered acceptable given Israel's size: ground transport to the ETC could be expected to take less than four hours. The different hospital roles were reflected in the differential distribution of PPE, intended to last only for a few hours in all hospitals except the ETC, which was equipped for a lengthy hospitalisation.

Magen David Adom (MDA) is the main emergency transport service in Israel, with extensive experience in all types of emergency evacuations. MDA designated and trained specific teams for each district as the sole personnel authorised to transport patients with suspected or confirmed EVD. The teams used a different PPE from other caregivers, with advanced chemical and biological protection including powered air purifying respirators (PAPR), because they were already familiar with this equipment. They were further equipped with negative-pressure patient transport units.

Patient samples were tested for EVD by PCR at a national-level high-safety laboratory. A detailed protocol for the safe collection, packaging and transport of specimens was issued [14].

Travellers from affected countries arriving in Israel were identified at air and sea borders both voluntarily, using informative posters and leaflets, and by border control officers reviewing passport logs. Travellers' temperatures were taken with a non-touch thermometer by personnel wearing gloves and a face shield. Symptomatic travellers were to be interviewed by airport medical staff and transported to the ETC. No symptomatic travellers have so far been identified by these controls. District public health offices were responsible for conducting follow-up of asymptomatic travellers twice a day for 21 days, and for epidemiological investigation of symptomatic cases and contact tracing, in a similar way as described by CDC [26]. Public health officers were instructed to minimise physical contact with suspected cases with the help of distance-enabling technologies (intercoms, video cameras, etc.). 
The PHS were responsible for executing the preparedness plan on the national level, by distributing updated guideline to the medical community, border control, other emergency response organisations and the general population, and by enlisting professional advice and assistance from the EMT and experts around the country as needed [14].

\section{Assessment of suspected cases in Israel}

Between October 2014 and February 2015, 80 asymptomatic travellers were identified and followed; none of them developed symptoms. However, some travellers arriving from West Africa circumvented border screening by avoiding public health staff and presenting themselves using a passport with no documentation of presence in the affected countries. Three febrile patients were assessed for suspected EVD, one before and two after the national guidelines became available. The latter two had evaded border identification and hence were not actively followed by PHS before presenting to the hospital. All three tested negative for Ebola virus and were diagnosed with other infectious diseases.

\section{Healthcare worker training}

Maintaining HCW safety was a cornerstone of the preparedness plan. Events in Africa, the US and Spain indicated that even excellent PPE is worthless without effective donning and doffing techniques and patient care practices. Therefore, the $\mathrm{MOH}$ instigated a crash programme to familiarise HCWs with PPE and infection control principles. These measures were aimed at building up confidence among HCWs, alleviating stress and easing any reluctance to participate in EVD patient care. The main topics covered were risk assessment for occupational EVD exposure, stringent contact precautions, proper PPE donning and doffing and vital steps in dealing with a patient with suspected EVD. Ninety briefings for hospitals, law enforcement and border control personnel were held by $\mathrm{MOH}$ staff, aided by military personnel qualified in PPE instruction. Briefings were followed by hands-on training. Proficiency was tested in 30 surprise drills, conducted at least once in every acute care hospital. These short drills simulated the admission of a suspected patient to the emergency department, followed by rapid assessment and transportation to the ETC. MOH staff evaluated adherence to the national guidelines and infection control practices. Media representatives were actively invited to participate in order to emphasise publicly the preparation efforts.

\section{Discussion}

Epidemics require complex responses. Although there are many similarities in the response plans for different diseases, no one generic plan can answer all eventualities. The recent EVD epidemic was characterised by a high fatality rate, lack of effective treatment or vaccine, and unclear mode of transmission. All of these led to anxiety among the general public and the healthcare community. Accordingly, the level of preparation and precautions taken in countries outside Africa was unprecedented.

Israeli health authorities constructed a comprehensive preparedness programme in anticipation of EVD importation from Africa. Although the risk was perceived to be low, up to four patients including limited local transmission, the implications were profound. Several elements of the Israeli response were unique to the EVD epidemic: follow-up of inbound travellers, designation of specific transport teams and a single medical centre and the stringent application of PPE. Experience with a handful of suspected cases, later found to be negative, validated this exacting approach. Similar actions in the US and the United Kingdom later reinforced the designation of regional ETCs $[27,28]$.

International infection control recommendations call for extreme precautions to prevent contact transmission of EVD and for some airborne precautions during aerosol-generating procedures. According to the WHO, solid waste needs to be burned or buried and liquid waste drained into the general sewage [15]. Nevertheless, most countries in the developed world implemented even stricter procedures during the EVD epidemic, such as use of PAPR, isolation in negativepressure rooms, and chemical or physical inactivation of any waste. Some countries relied on existing highlevel isolation units (HLIU) [29-32], but others, including Israel, had no such capability [33]. Much of the isolation infrastructure in Israel was developed as part of the response to SARS, smallpox and other airborne diseases and was not optimally suited to the typical EVD patient who produces large amounts of liquid and solid waste. In addition, hospital administrators were reluctant to have patients with EVD on the same ward as other patients. As construction of a HLIU was impossible in the existing time frame, the $\mathrm{MOH}$ decided to construct a free-standing negative-pressure isolation unit using customised tents, combined with appropriate sanitary and waste disposal facilities. In this manner, patients could be maintained in a highly secluded area within reach of a hospital yet far from hospital personnel and the public. This approach proved practical, easy to develop and relatively inexpensive compared with HLIU construction. The $\mathrm{MOH}$ is currently contemplating the construction of a HLIU in Israel; until then, the ETC serves as a practical solution for the treatment of future patients with highly contagious and hazardous diseases.

Even with traveller surveillance and guidance, unexpected cases of EVD might present at any emergency department. Thus, the $\mathrm{MOH}$ found it necessary to prepare every hospital in the country. Prompted by findings from Europe that only $16 \%$ of hospitals that were not intended to admit EVD patients, and $46 \%$ of all admitting hospitals had undergone preparatory exercises [29], the $\mathrm{MOH}$ included a vigorous educational campaign in its plan. The programme included educational sessions, combined with hands-on practice and 
surprise drills, and was successfully delivered to all acute-care hospitals in Israel within a few weeks.

\section{Conclusion}

In summary, we describe the preparedness programme of the Israeli $\mathrm{MOH}$ in response to the threat of EVD importation during the 2014-15 epidemic in West Africa. Although no patient was diagnosed with EVD in Israel, training and treatment of suspected patients showed that the plan was effective and manageable. A national protocol that relies on one specialised treatment unit, together with a moderately low-cost and rapidly constructed isolation facility, enabled a high level of care under significant economic constraints. Israel's programme may provide a template for emergency infectious disease response in other geographically small countries.

\section{Acknowledgements}

The authors wish to thank Beth-El industries Ltd (Zikhron Yaakov, Israel) for its contribution to EVD preparedness in Israel.

\section{Conflict of interest}

None declared.

\section{Authors' Contributions}

All the authors had a prominent role in the construction of the Israeli preparedness programme. TBN drafted the article. All authors participated in manuscript writing and revision. All authors read and approved the final manuscript.

\section{References}

1. World Health Organization (WHO). Ebola situation report, 21 October 2015. Geneva, WHO; 2015. p. 1-16. Available from: http://apps.who.int/ebola/current-situation/ ebola-situation-report-21-october-2015

2. European Centre for Disease Prevention and Control (ECDC). Medical evacuations. Stockholm: ECDC. [Accessed: $110 \mathrm{Ot}$ 2015]. Available from: http://ecdc.europa.eu/en/healthtopics/ ebola_marburg_fevers/Pages/medical-evacuations.aspx

3. European Centre for Disease Prevention and Control (ECDC). Italy confirms case of Ebola Virus Disease (EVD) in healthcare worker. Stockholm: ECDC; 2015. Available from: http:// ecdc.europa.eu/en/press/news/ layouts/forms/News DispForm.aspx?ID=1219\&List=8d b7286c-fe2d-476c-913318ff4cb1b568\&Source=http://ecdc.europa.eu/en/Pages/home. aspx

4. ChevalierMS, ChungW, SmithJ, WeilLM, HughesSM, JoynerSN, et al. Ebola virus disease cluster in the United States-Dallas County, Texas, 2014. MMWR Morb Mortal Wkly Rep. 2014;63(46):1087-8.PMID: 25412069

5. YacisinK, BalterS, FineA, WeissD, AckelsbergJ, PrezantD, et al. Ebola virus disease in a humanitarian aid worker - New York City, October 2014. MMWR Morb Mortal Wkly Rep. 2015;64(12):321-3.PMID: 25837242

6. KraftCS, HewlettAL, KoepsellS, WinklerAM, KratochvilCI, LarsonL, et al. The Use of TKM-100802 and Convalescent Plasma in 2 Patients With Ebola Virus Disease in the United States. Clin Infect Dis. 2015;61(4):496-502. DOI: 10.1093/cid/ civ334 PMID: 25904375

7. LyonGM, MehtaAK, VarkeyJB, BrantlyK, PlylerL, McElroyAK, et al. . Clinical care of two patients with Ebola virus disease in the United States. N Engl J Med. 2014;371(25):2402-9. DOI: 10.1056/NEJMoa1409838 PMID: 25390460
8. McCarthyM. Surgeon from Sierra Leone treated for Ebola in Nebraska dies.BMJ. 2014;349(nov18 4):g6942. D0I: 10.1136/ bmj.g6942 PMID: 25406131

9. LópazMA, AmelaC, OrdobasM, Dominguez-BerjonMF, ÁlvarezC, MartínezM, et al. First secondary case of Ebola outside Africa: epidemiological characteristics and contact monitoring, Spain, September to November 2014. Euro Surveill. 2015;20(1):21003. DOI: $10.2807 / 1560-7917 . E S 2015.20 .1 .21003$ PMID: 25613651

10. Bogochll, CreatoreMI, CetronMS, BrownsteinJS, PesikN, MiniotaJ, et al. Assessment of the potential for international dissemination of Ebola virus via commercial air travel during the 2014 west African outbreak. Lancet. 2015;385(9962):29-35. DOI: 10.1016/S0140-6736(14)61828-6 PMID: 25458732

11. WHO Ebola Response Team,. Ebola virus disease in West Africa--the first 9 months of the epidemic and forward projections.N Engl J Med. 2014;371(16):1481-95. DOI: 10.1056/ NEJMoa1411100 PMID: 25244186

12. Centers for Disease Control and Prevention (CDC). Case definition for Ebola virus disease (EVD). Atlanta: CDC. [Accessed: 12 Jun 2015]. Available from: http://www.cdc.gov/ vhf/ebola/hcp/case-definition.html

13. European Centre for Disease Prevention and Control (ECDC). Ebola virus disease case definition for reporting in EU. Stockholm: ECDC. [Accessed:12 Jun 2015]. Available from: http://ecdc.europa.eu/en/healthtopics/ebola_marburg_fevers/ EVDcasedefinition/Pages/default.aspx

14. Public Health Services. Ebola Virus Disease Outbreak, updated instructions, December 2014. Jerusalem: Israel Ministry of Health; 2014. Available from: http://www.health.gov.il/hozer/ bz25_2014.pdf. Hebrew.

15. World Health Organization (WHO). Interim infection prevention and control guidance for care of patients with suspected or confirmed filovirus haemorrhagic fever in health-care settings, with focus on Ebola. Geneva: WHO. 2014. Available from: http://www.who.int/csr/resources/publications/ebola/ filovirus_infection_control/en/

16. Centers for Disease Control and Prevention (CDC). Infection prevention and control recommendations for hospitalized patients under investigation (PUIs) for Ebola virus disease (EVD) in U.S. hospitals. Atlanta: CDC. [Accessed: 25May 2015] Available from: http://www.cdc.gov/vhf/ebola/healthcare-us/ hospitals/infection-control.html

17. MacIntyreCR, ChughtaiAA, SealeH, RichardsGA, DavidsonPM. Respiratory protection for healthcare workers treating Ebola virus disease (EVD): are facemasks sufficient to meet occupational health and safety obligations? Int J Nurs Stud. 2014;51(11):1421-6. DOI: 10.1016/j.ijnurstu.2014.09.002 PMID 25218265

18. Centers for Disease Control and Prevention (CDC). Guidance on personal p;rotective equipment to be used by healthcare workers during management of patients with Ebola virus disease in us hospitals, including procedures for putting on (donning) and removing (doffing). Atlanta: CDC. [Accessed: 12 Jun 2015]. Available from: http://www.cdc.gov/vhf/ebola/hcp/ procedures-for-ppe.html

19. WeingartlHM, Embury-HyattC, NfonC, LeungA, SmithG, KobingerG. Transmission of Ebola virus from pigs to nonhuman primates.Sci Rep. 2012;2:811. DOI: 10.1038/srepoo811 PMID: 23155478

20. JaaxN, JahrlingP, GeisbertT, Geisbert), SteeleK, McKeeK, et al. Transmission of Ebola virus (Zaire strain) to uninfected control monkeys in a biocontainment laboratory. Lancet. 1995;346(8991-8992):1669-71. DOI: 10.1016/S01406736(95)92841-3 PMID: 8551825

21. PiercyTJ, SmitherSJ, StewardJA, EastaughL, LeverMS. The survival of filoviruses in liquids, on solid substrates and in a dynamic aerosol.J Appl Microbiol. 2010;109(5):1531-9.PMID: 20553340

22. JohnsonE, JaaxN, WhiteJ, JahrlingP. Lethal experimental infections of rhesus monkeys by aerosolized Ebola virus.Int J Exp Pathol. 1995;76(4):227-36.PMID: 7547435

23. BorioL, InglesbyT, PetersCJ, SchmaljohnAL, HughesJM, JahrlingPB, et al. Hemorrhagic fever viruses as biological weapons: medical and public health management. JAMA. 2002;287(18):2391-405. DOI: 10.1001/jama.287.18.2391 PMID: 11988060

24. Gesser-EdelsburgA, Shir-RazY, HayekS, Sassoni-Bar LevO. What does the public know about Ebola? The public's risk perceptions regarding the current Ebola outbreak in an as-yet unaffected country. Am J Infect Control. 2015;43(7):669-75. DOI: 10.1016/j.ajic.2015.03.005 PMID: 25920703

25. Steinbuch Y, Fredericks B. Texas Ebola hospital worker is now in quarantine on cruise ship. New York Post. New York, NY; 2014

26. Centers for Disease Control and Prevention (CDC). Interim U.S. Guidance for Monitoring and Movement of Persons with 
Potential Ebola Virus Exposure. Atlanta: CDC. [Accessed: 3

Nov 2015]. Available from: http://www.cdc.gov/vhf/ebola/pdf/

monitoring-and-movement.pdf

27. Centers for Disease Control and Prevention (CDC). Current Ebola treatment centers. Atlanta: CDC. [Accessed: 20 May 2015]. Available from: http://www.cdc.gov/vhf/ebola/ healthcare-us/preparing/current-treatment-centers.htm

28. UK Secretary of State for Health. Ebola epidemic in West Africa. London: UK Government Department of Health; 2014 Available from: https://www.gov.uk/government/speeches/ ebola-epidemic-in-west-africa

29. de JongMD, ReuskenC, HorbyP, KoopmansM, BontenM, ChicheJ, et al. Preparedness for admission of patients with suspected Ebola virus disease in European hospitals: a survey, AugustSeptember 2014. Euro Surveill. 2014;19(48):20980. DOl: 10.2807/1560-7917.ES2014.19.48.20980 PMID: 25496571

30. EUNID Working Group, BannisterB, PuroV, FuscoFM, HeptonstallJ, IppolitoG. Framework for the design and operation of high-level isolation units: consensus of the European Network of Infectious Diseases.Lancet Infect Dis. 2009;9(1):45-56. DOI: 10.1016/S1473-3099(08)70304-9 PMID: 19095195

31. Ippolito G, Brouqui P, Lauria FN, Fusco FM. Letter to the editor: Management of patients with Ebola virus disease in Europe: High level isolation units have a key role. Euro Surveill. 2014;19(50):18-9.

32. BrouquiP, PuroV, FuscoFM, BannisterB, SchillingS, FollinP, et al. Infection control in the management of highly pathogenic infectious diseases: consensus of the European Network of Infectious Disease. Lancet Infect Dis. 2009;9(5):301-11. DOI: 10.1016/S1473-3099(09)70070-2 PMID: 19393960

33. SchillingS, FuscoFM, De lacoG, BannisterB, MaltezouHC, CarsonG, et al. Isolation facilities for highly infectious diseases in Europe--a cross-sectional analysis in 16 countries. PLoS ONE. 2014;9(10):e100401. DOI: 10.1371/journal. pone.0100401 PMID: 25350843 